\title{
Safety light curtain system using a hemispherical mirror with multiple frequency-modulated light sources
}

\author{
Yusuke Kenjo $^{\mathrm{a}, *}$, Ryosuke Suzue ${ }^{\mathrm{b}}$, Kohei Miyata ${ }^{\mathrm{a}}$, Shiyuan Yang ${ }^{\mathrm{a}}$ \\ ${ }^{a}$ Kyushu Institute of Technology, Address, 1-1Sensui-cho, Tobata-ku, Kitakyushu, Fukuoka, JAPAN 804-8550 \\ ${ }^{\mathrm{b}}$ Suzuki Motor Corporation, Address, 300Takatuka-cho, Minami-ku, Hamamatu, Shizuoka, JAPAN 432-8611 \\ *Corresponding Author: kenjou@ boss.ecs.kyutech.ac.jp
}

\begin{abstract}
Light curtains are used as a method to detect intruders at a lot of places now. However, it is necessary to adjust the position of the light detecting element accurately in order to receive the irradiating laser light successfully. We propose a new type of Safety Light Curtain System that uses a hemisphere side mirror and LEDs in this research. The hemisphere side mirror can reflect light rays surrounding of $120^{\circ}$ in the vertical direction and $360^{\circ}$ in horizontal direction. When an LED is placed at a position that is higher than the hemisphere side mirror, it is possible to reflect the light with the hemisphere side mirror even if the LED is arbitrarily set up. When an intruder passes between the LED and the hemisphere side mirror, the light of LED is intercepted and the output voltage of the light detecting element decreases. It can be judged by the threshold output voltage whether an intruder passed between LED and the hemisphere side mirror or not. The threshold output voltage is set by the PSoC microcomputer. The light of LED is modulated to $10 \mathrm{kHz}$ in order to reduce the influence of the surrounding turbulence light.
\end{abstract}

Keywords : Safety light curtain, hemispherical mirror, non-accurate set up, arbitrarily set up, modulated light source

\section{Introduction}

Recently, the intruder detection system for contactless to aim to detect the invasion to the danger area and the invasion prohibition district is widespread. The most popular technique uses light ray which cannot be most easily influenced by usage environment, although there are various techniques used in Intruder Detection Systems ${ }^{[1]}$.
There is a light curtain in one of the intruder detection systems that use light. A light curtain system contains a source of light ray and a receiving optical device. The penetration type irradiates light directly from the light emission element to the light detecting element. The source light is condensed with lenses to a beam ray and reaches to the receiving optical device in a place away. The irradiated light is intercepted when the intruder passes between the source of light and the receiving optical device., and the output of the light detecting element decreases. The intruder can be detected by judging output of the light detecting element. However, when light is irradiated from the source of light to the light part of receiving, the person must adjust an optical axis to this intruder detection system. Because of the directivity of the light irradiated from the light emission element the spot of light ray is very small so that the optical axis must be strictly adjusted. Therefore, it is not possible to set up arbitrarily, and convenience is scarce. In addition, when the system is set up in an arbitrary place for use, the source of light and the receiving optical device would be moved, it is necessary to adjust the optical axis at each installation. Therefore, it is chiefly used in limited facilities such as factories and airports where the movement of the source of light and the receiving optical device is not needed, and generality is scarce. We propose a new system for light curtain using a hemispherical mirror in this research. In this system, we do not use high directivity light such as a LED, but low directivity light LED is used. The reflected light is irradiated to the light detecting element by using a hemisphere side mirror. The source of light can be turned to the receiving optical device at any arbitrary position by the hemisphere side mirror and one need not to adjust the optical axis like the existing intruder detection system. According to the characteristic of the source light and the hemisphere side mirror, the source light is set up 
irradiating to the hemisphere side mirror, and the receiving optical device can be set up arbitrarily within the range where the reflected light from the source can be received. In addition, even if the source of light and the receiving optical device are moved, it does not need to adjust an optical axis in every case in the light part of receiving because with a hemisphere side mirror. Thus, this system can solve an unsuitable point in an existing system though use by carrying was unsuitable for an existing system.

\section{Light curtain system with hemisphere side mirror}

\subsection{Principle and composition}

Figure 1 shows the general view chart of light curtain system. Red high-power LED is used for the source of light in this research. The directivity of red high-power LED is low. Figure 2 shows the outline chart of the hemisphere side mirror. The hemisphere side mirror can project surroundings of $\theta 1=360^{\circ}$ and $\theta 2= \pm 90^{\circ}$ against $Y$ axis by seeing light above. It is possible to reflect with the hemisphere side mirror without adjusting the light part of receiving even if the source of light is arbitrarily set up, and if the source of light is a position that is higher than the hemisphere side mirror, it is a photodiode and receiving optical according to the characteristic of the hemisphere side mirror becoming of it easiness to adjust the direction of the source of light by the characteristic of red high-power LED.

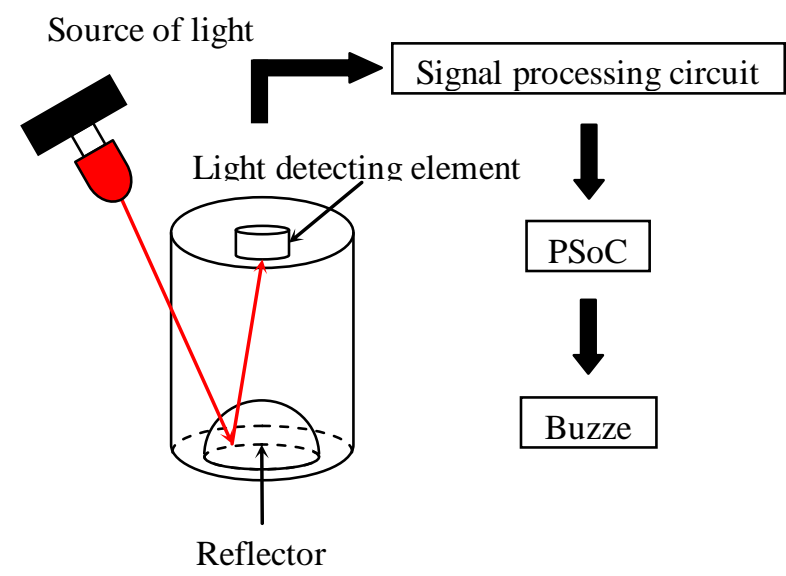

Fig 1. General view chart of light curtain system.

At this time, light from the source light is intercepted when an intruder passes between the source of light and the reflector (hemisphere side mirror), and the output voltage of the light detecting element (photodiode) decreases. When the output voltage becomes less than threshold, it is judged that the person passed between the source of light and the light part of receiving, and sounds the warning sound.

\section{$2.2 \quad$ Source of light}

In this research, light from the irradiating source is reflected by the hemisphere side mirror, and the reflected light is received by a light detecting element. The hemisphere side mirror must extract only the signal of the source of light to reflect the turbulence light. There, frequency modulation is carried out for the distinction between the source of light and the turbulence light. Because the turbulence light such as fluorescent lamps is low frequency, We have to modulate a source of light to frequency high enough. Then, the frequency of the source light is modulated to $10 \mathrm{kHz}$ in this research. The source light is modulated with a timer IC. Figure 3 shows the oscillation circuit that a timer IC was used (Refer to LMC555CN ). Figure 4 shows the oscillating wave pulse from the oscillation circuit. The frequency of this pulse wave is determined by the next equations, while the time intervals TH of on and TL of off are shown in Fig. 4.

$$
\begin{aligned}
& T_{L}=0.693\left(R_{1}+R_{2}\right) C_{1} \\
& T_{H}=0.693 \times R_{2} \times C_{1} \\
& f=\frac{1}{T_{H}+T_{L}}
\end{aligned}
$$




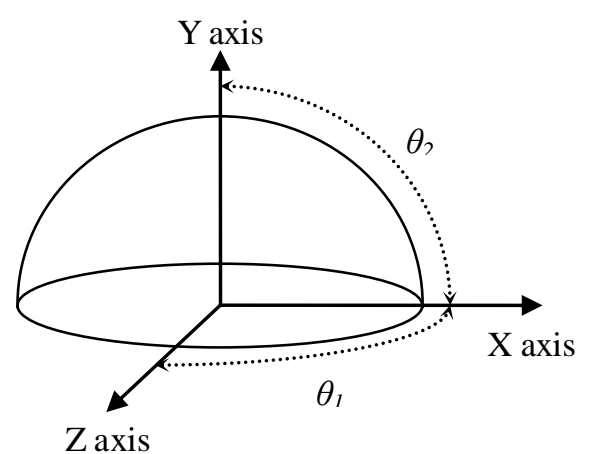

Fig 2. Outline chart of hemisphere side mirror.

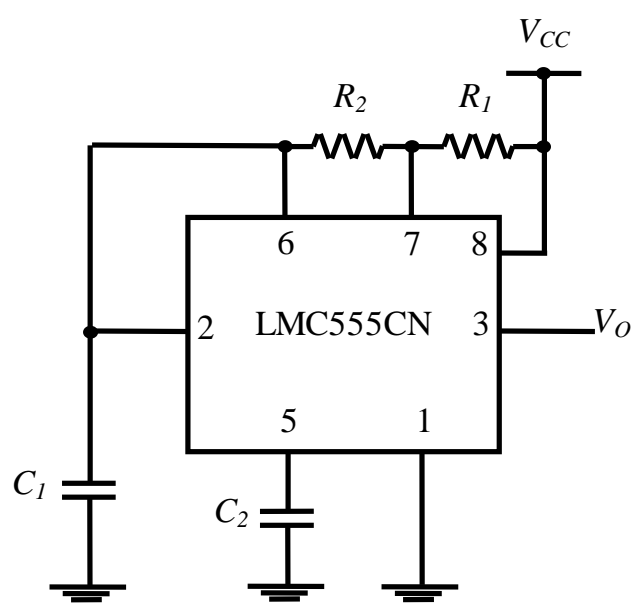

Fig 3. Frequency modulation circuit.

Parameters of the oscillation circuit can be determined by using Eqs. (2.1) (2.3). Timer IC outputs the wave pulse of $10 \mathrm{kHz}$ when uses $\mathrm{R} 1=450 \Omega$ and $\mathrm{R} 2=500 \Omega$ and $\mathrm{C} 1=10 \mathrm{pF}$ and $\mathrm{C} 2=10 \mathrm{uF}$.

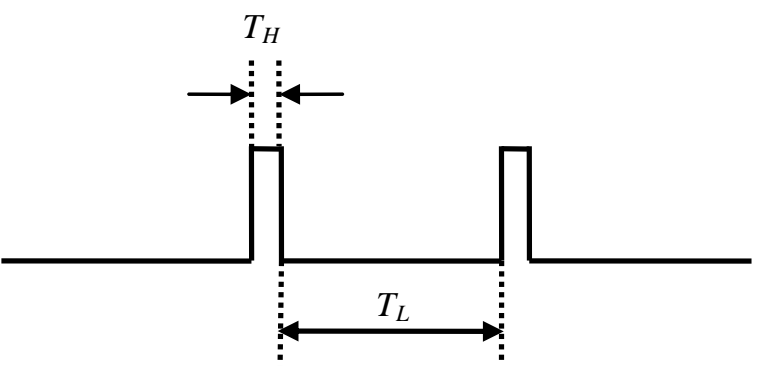

Fig 4. Oscillating wave pulse from frequency modulation circuit.

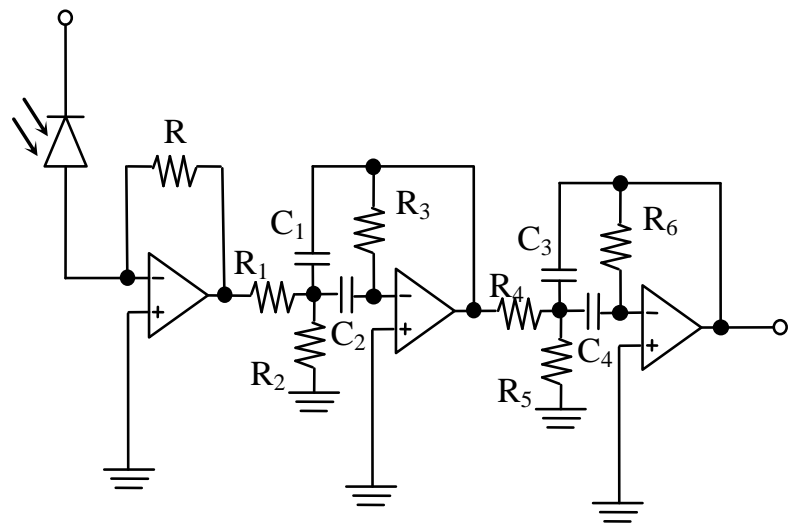

Fig 5. Circuit chart of signal processing.

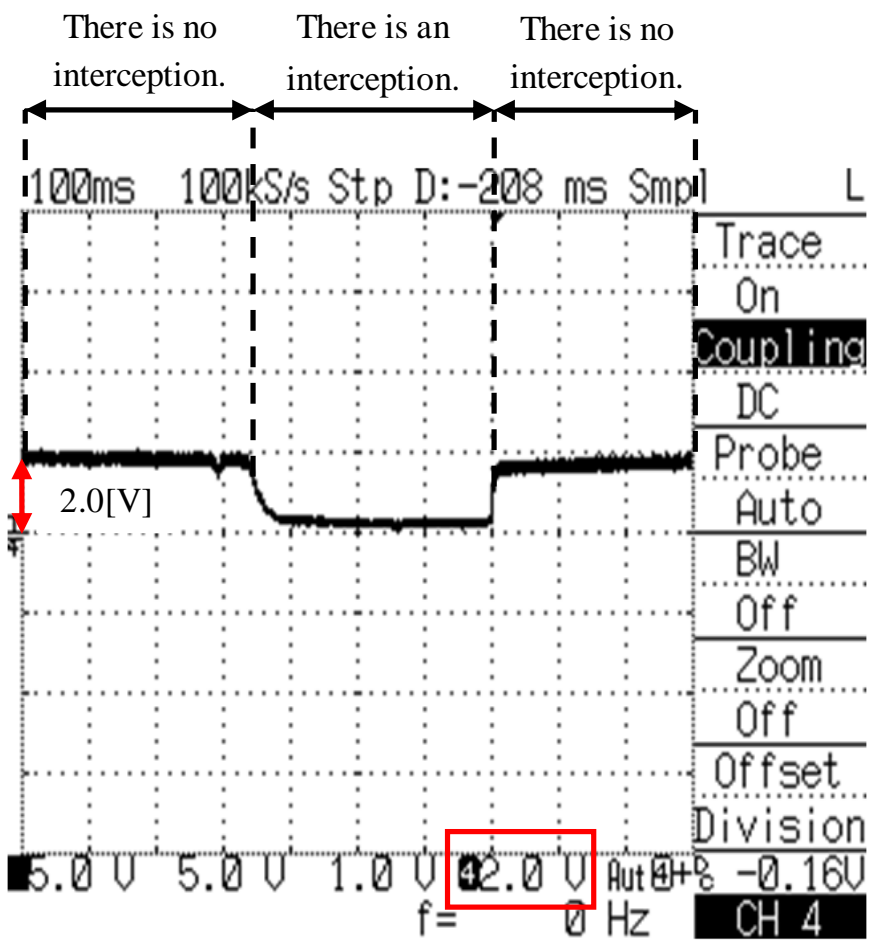

Fig 6. Output wave form when person passes between devices. 


\subsection{Signal processing circuit}

Figure 5 shows the circuit chart of the signal processing. By this signal processing circuit, current-voltage conversion is carried out and about $10 \mathrm{kHz}$ frequency component is the extracted by the current-filter circuit, and then the signal is amplified. Therefore, only the signal of the source light that is modulated to $10 \mathrm{kHz}$ can be extracted. The signal processing circuit uses $R=R_{7}=1 \mathrm{M} \Omega$ and $\mathrm{R}_{1}=\mathrm{R}_{4}=5.6 \mathrm{k} \Omega$ and $\mathrm{R}_{2}=\mathrm{R}_{5}=910 \Omega$ and $\mathrm{R}_{3}=\mathrm{R}_{6}=330 \mathrm{k} \Omega$ and $\mathrm{C}_{1}=\mathrm{C}_{3}=\mathrm{C}_{2}=\mathrm{C}_{4}=1 \mathrm{nF}$ and $\mathrm{C}_{5}=47 \mathrm{nF}$.

\subsection{Setting of threshold voltage}

A PSoC microcomputer is used in this research. A setup of the $\mathrm{A} / \mathrm{D}$ conversion and the threshold value is performed with a PSoC microcomputer, and a buzzer is sounded when PSoC judged that people passed between the light source and the receiving optical device. Figure 6 shows one example of the output wave form when the person passes between devices and the source of light is intercepted. In this research, invader detection is judged by change of output voltage. If people pass along between the light source and the receiving optical device, the light source is interrupted and output voltage decline.

It is judged whether a person passed between the light source and the receiving optical device or not, and the buzzer rings when the output voltage becomes less than threshold. However, because light from a light source cannot be completely intercepted even if people pass along between the source of light and the receiving optical device, output voltage does not become $0[\mathrm{~V}]$. Therefore, when the light source is interrupted, output has variation according to the distance of source of light to receiving optical device. Mistakes of judgment will occur when a constant threshold voltage is used. Therefore, we make experiments on the determination of threshold voltage in Sect. 3.2. As a result, it is understood that the output of receiving light from the source of light become $60 \%$ or less when a people intercepts light from the source of light. Little room is given in this research, and $70 \%$ is set as the threshold.

\subsection{Signal processing circuit by multiple frequency light sources}

Figure 7 shows the circuit chart of signal processing with multiple frequency-modulated light sources. Current-voltage conversion is carried out and then voltage components with $2.3 \mathrm{kHz}$ and about $10 \mathrm{kHz}$ are extracted by each current-filter circuit respectively, and each signal is amplified. Therefore, the light source signal with $2.3 \mathrm{kHz}$ and $10 \mathrm{kHz}$ can be obtained. The signal processing circuit uses $\mathrm{R}=1[\mathrm{M} \Omega], \quad \mathrm{R}_{1}=\mathrm{R}_{4}=5.6 \mathrm{k} \Omega$ and $\mathrm{R}_{2}=\mathrm{R}_{5}=910 \Omega$ and $\mathrm{R}_{3}=\mathrm{R}_{6}=330 \mathrm{k} \Omega$ and $\mathrm{R}_{7}=\mathrm{R}_{10}=15 \mathrm{k} \Omega$ and $\mathrm{R}_{8}=\mathrm{R}_{11}=110 \Omega$ and $\mathrm{R}_{9}=\mathrm{R}_{12}=470 \mathrm{k} \Omega$ and $\mathrm{C}_{1}=\mathrm{C}_{3}=\mathrm{C}_{2}=\mathrm{C}_{4}=1 \mathrm{nF}$ and $\mathrm{C}_{5}=\mathrm{C}_{7}=$ $\mathrm{C}_{6}=\mathrm{C}_{8}=0.01 \mathrm{uF}$.

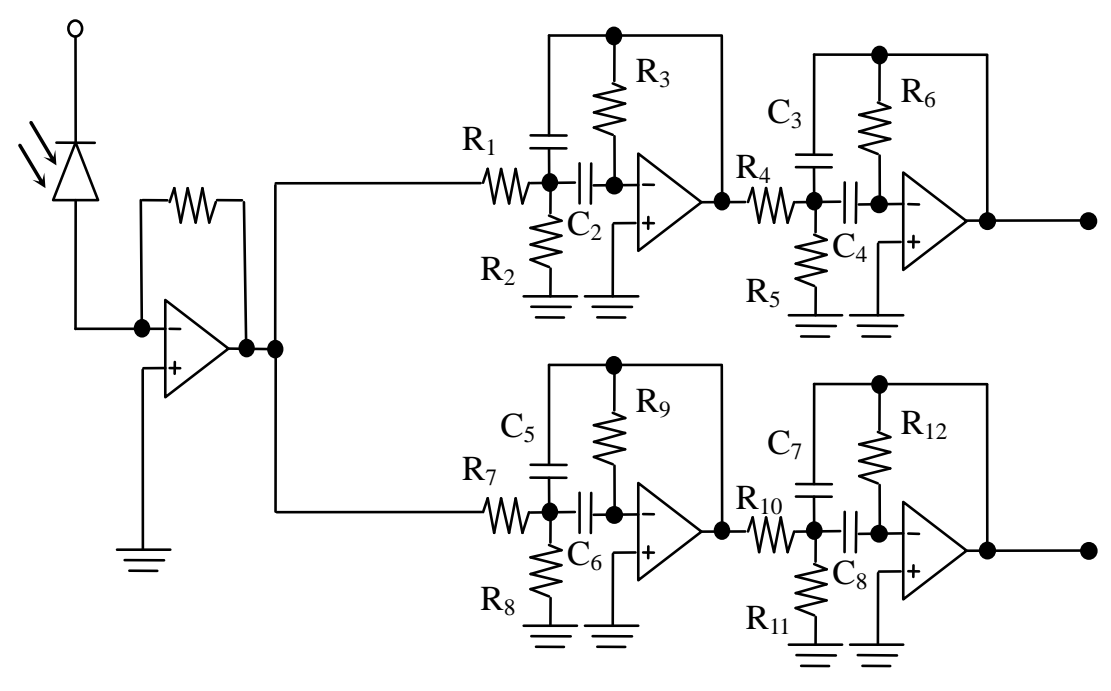

Fig 7. Circuit chart of signal processing with multiple frequency-modulated light sources. 


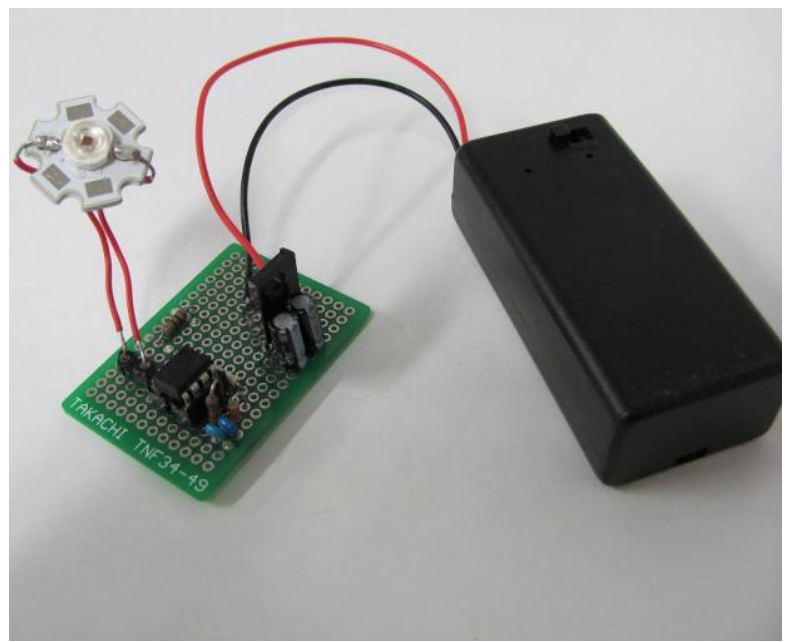

Fig 8. Source of light.

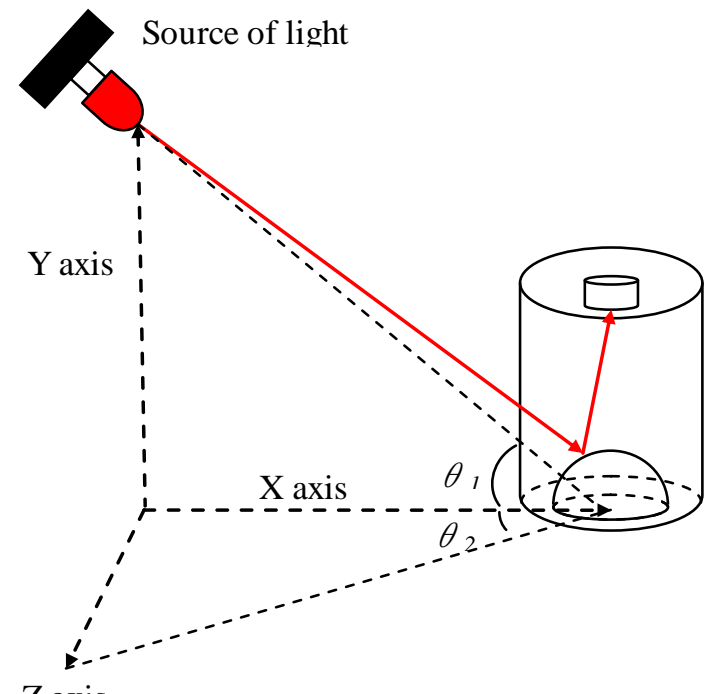

Fig 9. Measurement axis XYZ.

\section{Experiments}

\subsection{Device composition}

Figs 8 and 9 show the photograph of the source light and the receiving optical device produced in this research. The receiving optical device is $75 \mathrm{~mm}$ in length, and $85 \mathrm{~mm}$ in width, and $155 \mathrm{~mm}$ in height. Such a device is used and determination of the threshold and verification of operation were carried out.

\subsection{Device of threshold}

In the experiment, ratio is defined as the output when a people intercept light from the source of light to the output when light from the source of light is received directly.

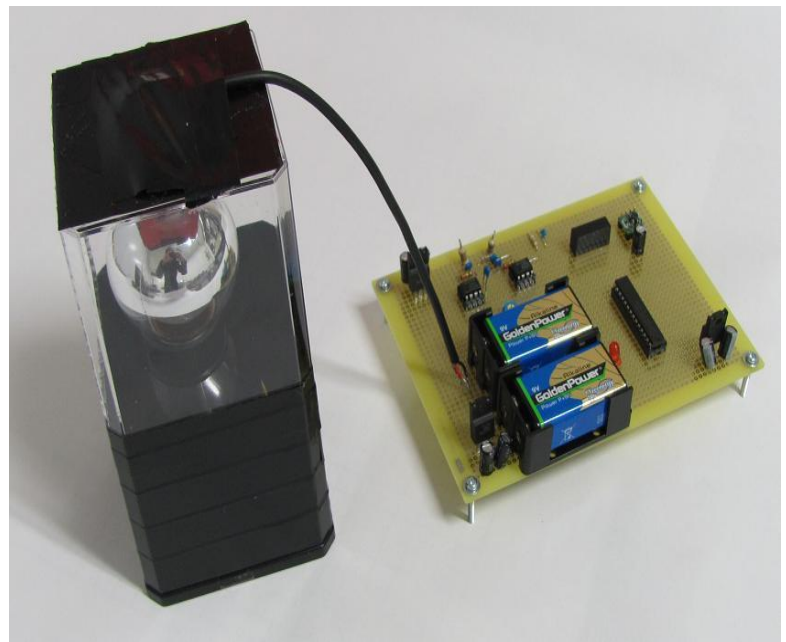

Fig 10. Light part of receiving.

Table 1. Ratio when intercepting it based on non-intercepting source of light.

\begin{tabular}{|c|c|c|c|c|c|}
\hline distance & $1[\mathrm{~m}]$ & $2[\mathrm{~m}]$ & $3[\mathrm{~m}]$ & $4[\mathrm{~m}]$ & $5[\mathrm{~m}]$ \\
\hline darkroom & $54[\%]$ & $38[\%]$ & $43[\%]$ & $30[\%]$ & $29[\%]$ \\
\hline fluorescent & $57[\%]$ & $34[\%]$ & $60[\%]$ & $41[\%]$ & $33[\%]$ \\
\hline
\end{tabular}

Therefore, the output voltage of the photo-diode was measured by the existence of interception. Experiments are carried out in two environments of under the darkroom and the fluorescent lamp. Figure 9 shows the measurement directions of $\mathrm{X}$ axis, $\mathrm{Y}$ axis, and $\mathrm{Z}$ axis. The experimental environment was assumed that the line between source of light and receiving optical device is at an angle of degrees to $\mathrm{X}$ axis in the $\mathrm{XY}$ plane and at an angle of $\theta 2$ degrees to $\mathrm{X}$ axis in the XZ plane. $\theta 1$ is measured to every $15\left[^{\circ}\right]$ in the range of $75\left[^{\circ}\right]$ from $0\left[^{\circ}\right]$, and $\theta 2$ is measured to every $30\left[^{\circ}\right]$ in the range of $180\left[^{\circ}\right]$ from $0\left[^{\circ}\right]$, and distance is measured to every $1[\mathrm{~m}]$ in the range of $5[\mathrm{~m}]$ from $1[\mathrm{~m}]$.

Table 1 shows the ratio on intercepting it based on non-intercepting the source of light. The maximum ratio is $60 \%$ from Table 1. It is assumed $70 \%$ to be a threshold more enough than $60 \%$ because it can avoid the mistakes 
judgement $^{[3]}$.

Source of light

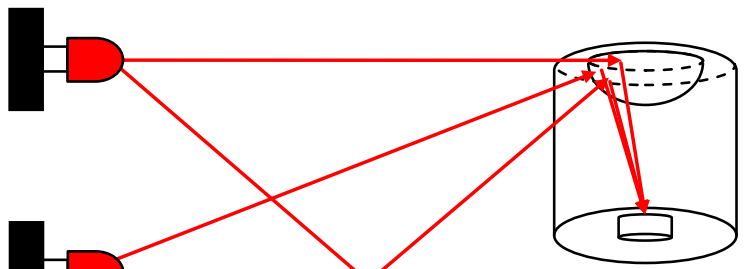

Light part of receiving



Fig 11. The intruder detection system in two or more sources of light and two or more light part of receiving.

Table 2 Recognition rate of intruder detection

\begin{tabular}{|c|c|c|}
\hline environment & darkroom & fluorescent \\
\hline recognition rate & $100[\%]$ & $100[\%]$ \\
\hline
\end{tabular}

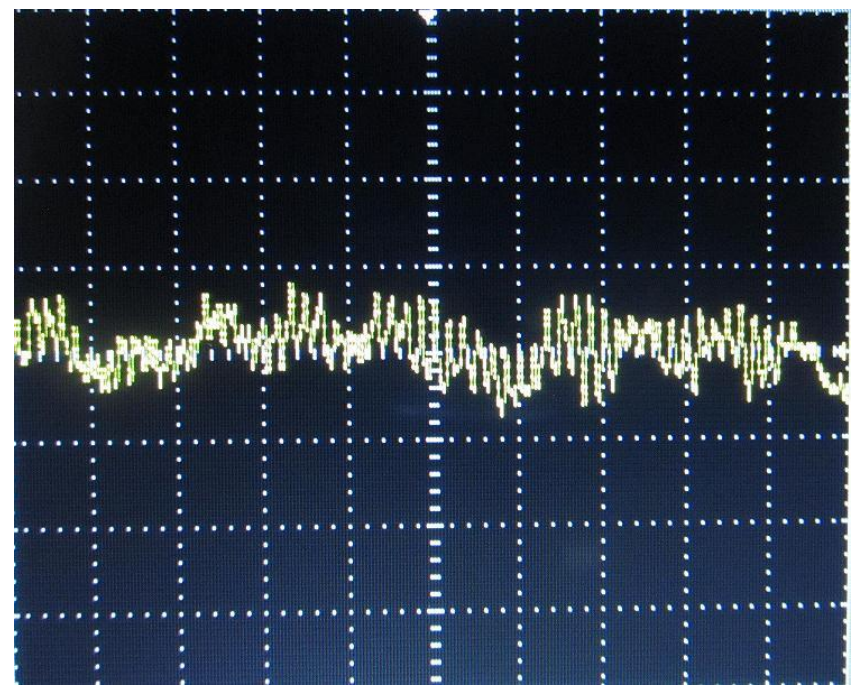

Fig 12. Signal before current-filter circuit.

\subsection{Operation verification of intruder detection}

A person passes between the source of light and the light receiving optical device after the setting of the threshold by the PSoC microcomputer ends, light from the source of light is intercepted, and whether the intruder detection operates normally is verified.

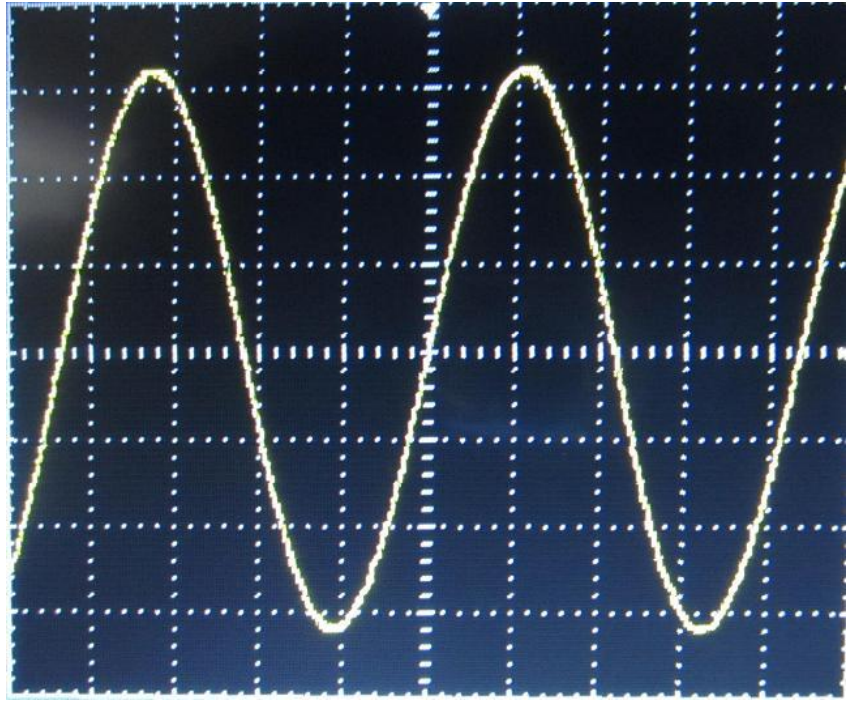

Fig 13. Signal of $2.3[\mathrm{kHz}]$.

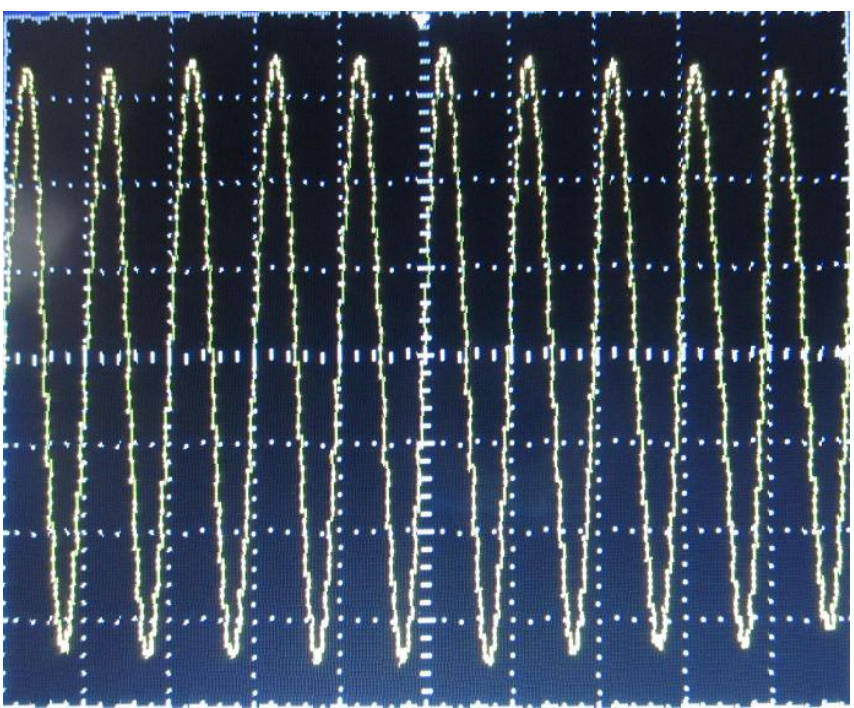

Fig 14. Signal of $10[\mathrm{kHz}]$.

Each ten operation verification was performed in the same angle as Section 3.2, distance, and an experiment environment, and it was checked whether the invasion detection had operated normally. Table 2 shows the recognition rate of the intruder detection by each distance as a verification result under each environment. The recognition rate of the intruder detection was $100 \%$ in all environments under the darkroom and the fluorescent lamp as understood from Table 2. The intruder detection system works normally from the verification result even if it arbitrarily is set up if the distance of source of light- light receiving optical device is $1 \mathrm{~m}-5 \mathrm{~m}$. 


\subsection{Measurement of multiple frequency light sources}

In the experiment, when turn on the multiple frequency-modulated light sources, then signals are extracted with each filter and their voltages are measured. Experiments are carried out under the condition of fluorescent lamp. The multiple frequency-modulated light sources are measured with $2.3[\mathrm{kHz}]$ and $10[\mathrm{kHz}]$. Distance is set at $1[\mathrm{~m}]$. Figure 11 shows the signal before current-filter circuit, and Fig. 12 show the signal of $2.3[\mathrm{kHz}]$, and Fig. 13 show the signal of $10[\mathrm{kHz}]$. It is possible to extract the signal of $10[\mathrm{kHz}], 2.3[\mathrm{kHz}]$ from Fig. 11, Fig. 12, Fig. 13. Therefore, because multiple light sources are available, set up as Fig. 14, it is possible to improve both the detection range and detection accuracy. In addition, distinction of the optical axis is possible, so it is possible to determine the location and size of the intruder.

\section{Conclusions}

In this research, we proposed the safety light curtain system for the intruder detection with red high-power LED, a photodiode, and a hemispherical mirror. As a conclusion, the result achieved from this research is shown below:

(1) If the source of light and the light receiving optical device are position in which light from the source of light can be reflected with the hemisphere side mirror, it is possible to operate as a normal system in an arbitrary place.

(2) It is possible to operate as a system that it is normal without a detailed adjustment of the source light and the light detecting element like the existing system, just to turn the source of light to the light part of receiving.

(3) The intruder detection system with two or more sources of light works correctly. Detection accuracy by two or more source of light and receiving optical device will be made as an improvement.

(4) Distinction of the optical axis is possible, so it is possible to determine the location and size of the intruder.

(5) When the existing system sets up the device, the installation is difficult, and it takes time in the amateur. But everyone can arbitrarily set it up widely using this system from the result of (1) and (2).
Therefore, the safety light curtain with a hemispherical mirror can be achieved. The improvement of convenience and generality was shown because it was possible to set it up widely and arbitrarily with low directivity LED and hemispherical mirror.

Future tasks include the following:

- Reduction of influence of turbulence light by accuracy improvement of band-pass filter.

\section{References}

[1] Keyence Corporation, "Safety light curtain safety guide book" (2009-2010).

[2] Naoki Midorikawa, "Development of the Online Simulation for Displaying of Wide Field of View Images using Small Size Multi Camera System", Robotics and Mechatronics lecture meeting, 2P2-B04,2008.

[3] Yusuke Kenjo, Ryosuke Suzue, Huimin Lu, Kohei Miyata, Shiyuan Yang, Seiichi Serikawa "A novel safety light curtain system using a hemispherical mirror", SPIE. 8561, 2012. 